\title{
Effects of stress and sex on acquisition and consolidation of human fear conditioning
}

\author{
Michael Zorawski, ${ }^{1,3}$ Nineequa Q. Blanding, ${ }^{1}$ Cynthia M. Kuhn, ${ }^{2}$ and Kevin S. LaBar ${ }^{1,4}$ \\ ${ }^{1}$ Center for Cognitive Neuroscience, Duke University, Durham, North Carolina 27708, USA; ${ }^{2}$ Department of Pharmacology and \\ Cancer Biology, Duke University Medical Center, Durham, North Carolina 27708, USA; ${ }^{3}$ Department of Psychology, National \\ University of Singapore, 117570, Singapore
}

\begin{abstract}
We examined the relationship between stress hormone (cortisol) release and acquisition and consolidation of conditioned fear learning in healthy adults. Participants underwent acquisition of differential fear conditioning, and consolidation was assessed in a 24-h delayed extinction test. The acquisition phase was immediately followed by an 11-min psychosocial stress period (arithmetic test combined with a public speech). Salivary cortisol was sampled at various time points before and after acquisition and retention of fear conditioning. Results showed two effects of endogenous cortisol. Post-acquisition cortisol correlated with fear acquisition in male but not female participants. In addition, post-acquisition cortisol correlated with consolidation of fear but only in those participants with high cortisol levels. We conclude that in the short term, a robust and sexually dimorphic relationship exists between fear learning and stress hormone levels. For those participants whose fear learning is accompanied by high stress hormone levels, a long-term relationship exists between cortisol release and memory consolidation. These short-term and long-term effects may relate to the differential involvement of mineralocorticoid and glucocorticoid receptor subtypes, respectively. The findings have implications for understanding the role of stress, sex, and hormones in different stages of fear learning and memory.
\end{abstract}

Fear conditioning constitutes an adaptive cognitive mechanism in most organisms, allowing them to effectively learn about danger-relevant relationships in the environment and thus enhancing their survival chances. In a typical fear conditioning procedure in the laboratory, a previously neutral conditioned stimulus (CS) predicts the occurrence of an aversive unconditioned stimulus (US) and, as a result, acquires emotional properties. Conditioning is established when the CS triggers a conditioned response in the form of arousal reactions. An increased release of adrenocortical stress hormones-glucocorticoids-through activation of the hypothalamic-pituitary-adrenal (HPA) axis represents an important physiologic component of such arousal responses to fearful stimuli. The principal glucocorticoid in humans is cortisol.

Basal and stress levels of glucocorticoids vary across individuals (Piazza et al. 1991; Meaney et al. 1993; Cools and Gingras 1998; Kirschbaum et al. 1999; Kabbaj et al. 2000; Stone et al. 2001; Bartels et al. 2003; Rohleder et al. 2003; Steptoe et al. 2003) and play an important but complex role in learning and memory (McEwen and Sapolsky 1995; Lupien and McEwen 1997; Roozendaal 2003; Wolf 2003). While cortisol may be detrimental to memory function under some circumstances-i.e., when levels are elevated chronically (Starkman et al. 1992; Luine et al. 1993, 1994; Arbel et al. 1994; Bodnoff et al. 1995; Conrad et al. 1996; Newcomer et al. 1999; Park et al. 2001) or during memory retrieval (De Quervain et al. 1998, 2000; Buss et al. 2004) — they also have acute facilitative effects, presumably constituting part of the underlying neurobiological mechanism responsible for enhanced learning and memory of emotionally relevant material. Acute memory-enhancing effects of glucocorticoids may operate at two stages of memory processing, the initial acquisition (encoding) stage and the delayed consolidation stage. Positive rela-

\section{${ }^{4}$ Corresponding author.}

E-mail klabar@duke.edu; fax (919) 681-0815.

Article published online before print. Article and publication date are at http:// www.learnmem.org/cgi/doi/10.1101//m.189106. tionships between acute cortisol levels and immediate effects on learning and memory are reflective of the former, whereas relationships with delayed retention imply either the former or the latter, although selective consolidation effects are inferred when cortisol is manipulated after the memory encoding task has terminated.

Studies in both humans and experimental animals have found that high endogenous baseline levels or exogenous administration of cortisol or stress prior to a memory task is associated with better immediate performance, implying beneficial effects on memory acquisition or encoding (Wilson et al. 1975; Beckwith et al. 1986; Shors et al. 1992; Fehm-Wolfsdorf et al. 1993; Bemelsmans et al. 2002; Domes et al. 2002; Lupien et al. 2002a,b; Abercrombie et al. 2003; Akirav et al. 2004; Putman et al. 2004; Maheu et al. 2005b; Zorawski et al. 2005). However, the issue is not unequivocal, and contradictory results have been reported (Wolkowitz et al. 1990; Kirschbaum et al. 1996; Lupien et al. 1997, 1999; Wolf et al. 2001a,b; Maheu et al. 2004). The interaction of several variables may be responsible for the discrepant findings, including the proposed inverted U-shaped dose response function of glucocorticoids (Kovacs et al. 1977; Diamond et al. 1992; Lupien and McEwen 1997; Conrad et al. 1999), the distinct roles of different receptor types (Veldhuis et al. 1982; Oitzl and de Kloet 1992; Lupien and McEwen 1997; de Kloet 2004), the natural diurnal variation of endogenous cortisol levels (Fehm-Wolfsdorf et al. 1993; Lupien et al. 1999, 2002a; Maheu et al. 2005a), and procedural factors such as the timing of the retention test in relation to the learning phase. If the retention test is arranged temporally close to acquisition, possibly facilitative acquisition effects may be masked by the impairing effects on memory retrieval (De Quervain et al. 1998, 2000).

Hormonal manipulations affect individuals differently, and one source of across-subject variance is biological sex. (Shors et al. 1992) found that stress facilitated subsequent classical conditioning in male rats both $24 \mathrm{~h}$ later and immediately afterwards (Shors 2001), whereas detrimental effects were found in female 
rats (Wood and Shors 1998; Wood et al. 2001; Shors 2004). Corticosterone levels were correlated with performance in male, but not female rats (Wood et al. 2001), whose performance was related to their current stage of menstrual cycle. These findings prompted the authors to suggest that learning in males and females may be differentially affected by distinct hormonal systems and their interactions. In line with this idea, we have recently presented evidence for a sex-specific relationship between cortisol and human fear conditioning (Zorawski et al. 2005). Acquisition of conditioning was correlated with salivary cortisol levels in male but not female participants. It is possible that, in females, the relationship between cortisol and fear acquisition is further modulated by other hormones such as estrogen. Additional support for this idea comes from a recent human study by Jackson et al. (2006), who found that psychosocial stress induced prior to conditioning enhanced fear acquisition in males but impaired fear acquisition in females. Finally, Wolf et al. (2001b) also reported a sex-specific relationship between exogenously manipulated cortisol levels and memory performance in males but not females.

In addition to their immediate role in memory acquisition, glucocorticoids are thought to facilitate memory consolidation as well. Evidence for this function of stress hormones comes mainly from conditioning studies in animals (Flood et al. 1978; Oitzl and de Kloet 1992; Sandi and Rose 1994; Roozendaal and McGaugh 1996; Zorawski and Killcross 2002, 2003; Hui et al. 2004; for reviews, see Roozendaal 2003; McGaugh 2004) and declarative memory experiments in humans (Buchanan and Lovallo 2001; Abercrombie et al. 2003; Cahill et al. 2004; Maheu et al. 2004). In these studies, manipulation of stress hormone levels tends to facilitate retention of memories following a delay, which implicates an effect on consolidation processes. To our knowledge, the only study to have investigated the role of stress hormones in the consolidation of classical conditioning in humans was carried out in our own laboratory (Zorawski et al. 2005) but found no significant effects.

Glucocorticoids bind to two types of receptors: the lowthreshold high-affinity mineralocorticoid receptors (MRs), which are largely occupied by endogenous glucocorticoids under basal conditions, and the high-threshold low-affinity glucocorticoid receptors (GRs), which primarily get activated under stress or during the circadian peak (Reul and de Kloet 1985; de Kloet et al. 1993; Reul et al. 2000). It has been suggested that the two receptor types subserve different cognitive functions (Lupien and McEwen 1997; Oitzl et al. 1997). MRs are thought to mediate nonspecific aspects of memory acquisition, such as sensory integration and selective attention (Oitzl and de Kloet 1992; Sandi and Rose 1994; Lupien and McEwen 1997; Oitzl et al. 1997; Lupien et al. 2002a) and thus may be responsible for the immediate, facilitative effects of cortisol on memory acquisition (Wood et al. 2001; Zorawski et al. 2005). The sex-specificity of this relationship may be due to estrogen effects on MR affinity and availability (Wood et al. 2001). GRs, in contrast, have been implicated in memory consolidation processes (Oitzl and de Kloet 1992; Sandi and Rose 1994; Zorawski and Killcross 2002; Roozendaal 2003). In our previous fear conditioning study (Zorawski et al. 2005), we argued that the failure to find a relationship between stress hormone release and 24-h consolidation of fear memory was likely due to the fact that we analyzed nonmanipulated endogenous hormone levels on a moderately arousing task which may not have been high enough to sufficiently engage GRs in a majority of healthy participants. Not much is known about sex-specificity of putative GR-related effects of cortisol on memory consolidation in humans. Some declarative memory experiments in support of GR-mediated modulation of memory consolidation by stress and glucocorticoids have employed both male and female participants (Buchanan and Lovallo 2001; Cahill et al. 2004), suggesting that this relationship may exist independently of sex.

The current study pursued two goals. First, it aimed to replicate our recent findings of a sex-specific relationship between endogenous cortisol levels and acquisition of human fear learning in males but not females (Zorawski et al. 2005). Secondly, it sought to further elucidate the relationship between cortisol levels and consolidation of fear learning. Healthy adults underwent differential delay conditioning using fear-relevant CSs and a shock US while skin conductance responses (SCRs) were recorded. In contrast to our previous report (Zorawski et al. 2005), the current study employed a psychosocial stress induction procedure immediately after conditioning to experimentally elevate post-acquisition cortisol levels and putatively engage GRs. Stress was induced by administration of the paced auditory serialaddition test (PASAT), a standard stress battery consisting of mental arithmetic tasks (see Materials and Methods), followed by a 4-min public speech in which the participant reflected on his or her performance on the PASAT and provided a self-assessment of math ability. To distinguish the contribution of stress hormones to acquisition versus consolidation processes, a 24-h delayed extinction test was conducted as an assessment of fear retention. Salivary cortisol samples were obtained at baseline and at several points following acquisition training and the extinction test. Conditioning data were analyzed as a function of cortisol levels and sex using both group-averaged and individual-difference analysis strategies.

Consistent with animal models and our prior report (Zorawski et al. 2005), we hypothesized a replication of our finding of a sex-specific positive correlation between post-acquisition cortisol levels and conditioned fear acquisition (in males but not females). This result would indicate a sexually dimorphic relationship between stress hormone release and immediate fear learning. Second, we hypothesized that post-acquisition stress hormones would positively correlate with 24-h consolidation of fear memories. That is, individual variability in stress hormone levels taken after fear acquisition/stress induction should predict which individuals show better retention of fear $24 \mathrm{~h}$ later. Based on previous research in animals (Oitzl and de Kloet 1992; Sandi and Rose 1994; Zorawski and Killcross 2002; Roozendaal 2003), consolidation effects depend on stress hormone levels being high enough to engage GRs. Accordingly, we predicted that the positive relationship between post-acquisition cortisol and memory consolidation would be evident only in individuals with high stress levels following training/stress induction. Given the limited existing literature regarding this issue, we made no a priori prediction about possible sex-specific effects in fear memory consolidation.

\section{Results}

\section{Conditioned responding}

Conditioning data from Day 1 were analyzed by a four-way mixed ANOVA, with Sex as a between-group factor (female, male) and CS type (CS+, CS - ), Phase (habituation, acquisition), and Block (early, late) as repeated measures (Fig. 1). There was a significant interaction of CS type (CS+, CS - ) and Phase (habituation, acquisition), $F_{(1,33)}=7.13, P<0.02$. Simple effects analysis revealed greater responding to the $\mathrm{CS}+$ than the $\mathrm{CS}-$ in acquisition, $F_{(1,33)}=10.06, P<0.01$, but not during habituation, $F<1$, which reflects successful fear learning. There were no significant main effects or interactions involving Sex (all Ps $>0.05$ ), suggesting similar conditioning levels in females and males.

To examine whether conditioned responding was retained over a 24-h delay, a planned contrast analysis was carried out in the form of a three-way mixed ANOVA with Sex (female, male) as a between-groups factor, and CS type (CS+, $\mathrm{CS}-$ ) and Phase (late 


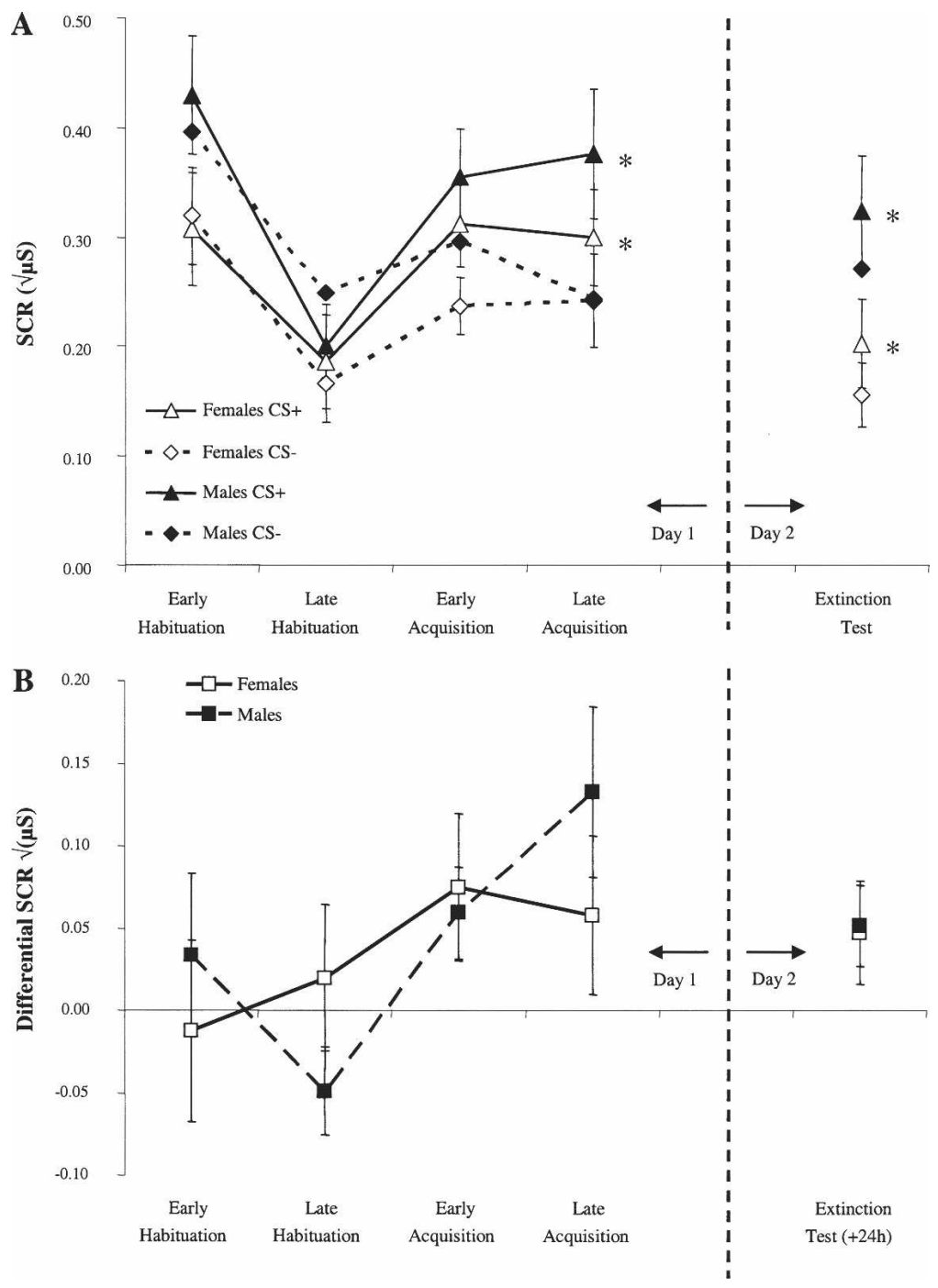

Figure 1. $(A)$ Mean skin conductance responses (SCR) to the reinforced $C S+$ and the non-reinforced $\mathrm{CS}-$ in females and males across different phases of conditioning and the 24-h delayed retention test. ${ }^{*} P<0.02(C S+$ vs. CS - ). (B) Differential SCR expressed as the difference between responses to the CS+ and $\mathrm{CS}-. \mu \mathrm{S}=$ microSiemens. Error bars reflect SEMs.

habituation, late acquisition, extinction) as repeated measures. This analysis focused on the late block trials in the habituation and acquisition phases since we were interested in comparing performance on the retention test to that obtained at the end of habituation and acquisition training. A significant CS type by Phase interaction was found, $F_{(1,33)}=4.77, P<0.02$. Simple effects analysis revealed greater responding to the CS+ than to the CS - in late acquisition, $F_{(1,33)}=6.87, P<0.02$, and extinction, $F_{(1,33)}=6.40, P<0.02$, but not in habituation, $F<1$. The factor Sex was not significant either as a main effect or as an interaction with the other variables ( $P$ S $>0.05)$.

\section{Unconditioned responding}

SCRs to the US did not differ between female $(M=1.02$, $S D=0.33)$ and male $(M=1.06, S D=0.51)$ participants $(t<1)$, which shows equivalent unconditioned responding to the shock as a function of sex.

\section{Subjective measures}

While males selected a higher voltage level of the shock than females (females, $M=64.67 \mathrm{~V}, S D=19.50$; males, $M=89.50 \mathrm{~V}$,
$\left.S D=15.72 ; t_{(1,33)}=-4.17, P<0.001\right)$, they did not differ in the perceived subjective irritation of the shock (females, $M=4.27, S D=1.28$; males, $M=3.75, S D=1.29 ; t_{(1,33)}=1.18$, $P=0.25)$. Females and males also did not differ in their perceived emotionality of the snake and spider images (females, $M=2.20, S D=1.32$; males, $M=2.00, S D=1.34 ; t<1$ ) nor in the perceived stressfulness of the study overall (females, $M=3.53, S D=1.77$; males, $M=2.95, S D=1.50$; $\left.t_{(1,33)}=1.05, P=0.30\right)$. Female participants reported higher levels $(M=2.83$; $S D=0.80)$ of perceived stress between sessions than males $(M=2.26$; $S D=0.76$ ) as measured by the Daily Stress Inventory (DSI; see Materials and Methods), $t_{(1,33)}=2.17, P<0.04$.

\section{Conditioning, cortisol, and sex}

Behaviorally, conditioning was best observed in the late phase of acquisition, as predicted. Given the temporal dynamics of saliva cortisol assessment (see Materials and Methods), the most appropriate time point to link endogenous cortisol to the late portion of acquisition training/stress induction was Sample 4, obtained about $45 \mathrm{~min}$ after the beginning of conditioning (and about $30-35 \mathrm{~min}$ after late acquisition). The relationship among sex, post-acquisition cortisol (Sample 4), and fear conditioning was analyzed in two ways-first, by median-split analysis (dividing participants into high vs. low stress groups according to cortisol values obtained from Sample 4) and second, by correlational analyses conducted on individual participant data. A three-way mixed ANOVA with Sex (female, male) and Cortisol group (high, low) as between-groups factors, and Phase (late acquisition, extinction) as a repeated measure revealed a significant three-way interaction, $F_{(1,40)}=6.80$, $P<0.02$ (see Fig. 2). Follow-up ANOVAs for each sex revealed a significant interaction of Cortisol group and Phase in males, $F_{(1,18)}=15.11, P<0.01$, but not in females. Simple-effects analysis revealed greater conditioned responding in high- than in lowcortisol males during late acquisition, $F_{(1,20)}=17.83, P<0.001$, but not in the 24 -h delayed retention test, $F<1$. In females, no differences were found between Cortisol groups in either phase of learning (late acquisition: $F<1$; extinction: $F_{(1,12)}=2.55$, $P=0.14)$. In line with these median-split results, a significant correlation between post-acquisition cortisol and differential conditioned responding during late acquisition was found in males, $r_{(18)}=0.57, P<0.01$, but not females, $r_{(13)}=-0.10$, $P=0.74$ (Fig. 3 ). There were no significant correlations between post-acquisition cortisol levels and conditioned responding in the delayed retention test in males, $r_{(18)}=0.19, P=0.42$, or females, $r_{(13)}=0.18, P=0.53$. A $2 \times 4$ mixed ANOVA with Sex (female, male) as between-group factor and Cortisol sample (1-4) as repeated measure revealed no significant main effect of Sex $(F<1)$ and no significant interaction, $F_{(3,87)}=2.06, P=0.21$, 


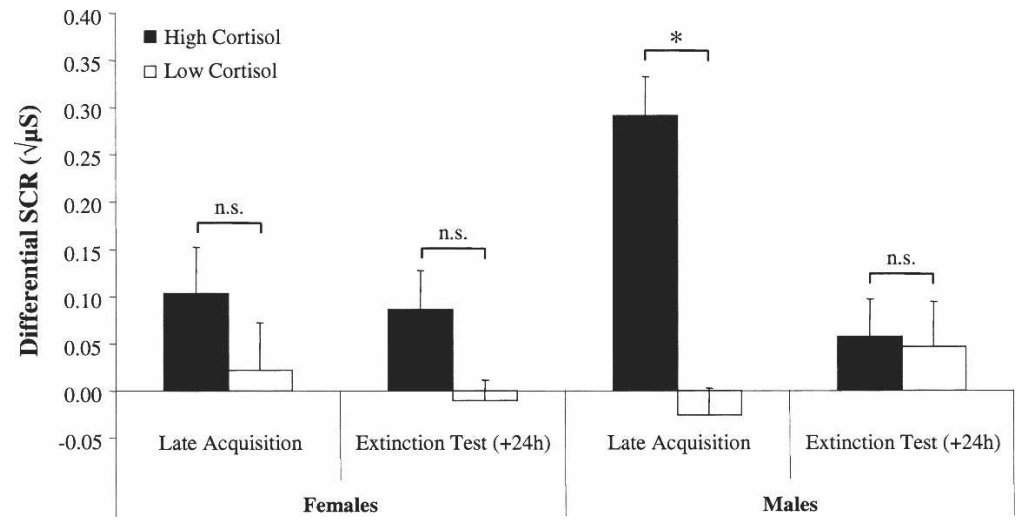

Figure 2. Differential skin conductance responses (SCRs), expressed as the difference between CS+ and CS-, during late acquisition and the 24-h delayed retention test in females and males as a function of cortisol level. The participants were assigned to high- and low-cortisol groups according to a median split of post-acquisition salivary cortisol levels $(+45 \mathrm{~min}) .{ }^{*} P<0.001 ;$ n.s. $=$ not significant. Error bars represent SEMs.

showing that there were no sex differences in baseline or postacquisition cortisol levels.

In conclusion, these data constitute a successful replication of our previous report (Zorawski et al. 2005), revealing a robust relationship between post-acquisition cortisol levels and conditioned fear acquisition in males but not females, but no relationship between cortisol levels and consolidation of fear learning as assessed during the 24-h delayed retention test in either sex.

\section{Verification of experimental stress manipulation on cortisol levels}

To assess whether the psychosocial stress procedure increased post-acquisition cortisol levels, cortisol data from this experiment were compared with those of our previous study (Zorawski et al. 2005). The procedure of that study was identical to the current one except that no psychosocial stress induction procedure was carried out after the acquisition phase of conditioning. It therefore served as a control condition. A three-way mixed ANOVA with Group (previous study, current study) and Sex (female, male) as between-groups factors, and Cortisol sample (1-4) as a repeated measure, was carried out (Fig. 4). Results showed a significant Group by Cortisol sample interaction, $F_{(3,213)}=3.86$, $P<0.01$. Simple effects analysis revealed that post-acquisition cortisol levels were significantly higher in the current study for Sample 4, $F_{(1,73)}=10.36, P<0.01$, but not for the other samples (all Fs $<1$ ). It is important to note that there were no baseline cortisol differences between experimental groups. The selective increase in cortisol taken at time point 4 for participants in the current study demonstrates that the psychosocial stress procedure increased post-acquisition cortisol levels overall.

\section{Cortisol, sex, and fear memory consolidation: Individual difference analysis of pooled data}

Although the psychosocial stress procedure reliably increased post-acquisition cortisol levels in the current study, these participants on average did not exhibit better consolidation as measured by 24 -h delayed retention. However, individual differences in endogenous cortisol levels may have interacted with the effects of the psychosocial stress procedure. To increase statistical power to observe a relationship between stress hormone levels and fear memory consolidation, we pooled data across our previous study (Zorawski et al. 2005) and the current study. Participants were divided into high- and low-cortisol groups by a median split of the pooled sample according to post-acquisition cortisol levels (Sample 4), and then Pearson correlation coefficients were conducted between post-acquisition cortisol levels and conditioned fear retention within each split group (Fig. 5). This procedure allows an analysis of the relationship between post-acquisition cortisol levels and consolidation of fear learning as a function of cortisol irrespective of the circumstances under which different cortisol levels occurred (e.g., endogenous cortisol levels for participants in Zorawski et al. [2005] vs. additional psychosocial stress induction in the current study). Fifty-six percent of participants in the high-cortisol group and $31 \%$ of participants in the low-cortisol group came from the current study. The analyses revealed a positive correlation between post-acquisition cortisol and conditioned fear retention in the highcortisol group, $r_{(37)}=0.35, P<0.04$, but not in the low-cortisol group, $r_{(37)}=-0.09, P=0.60$. In other words, we found a linear relationship between post-acquisition cortisol levels and 24-h delayed fear memory retention, but only for those participants exhibiting high stress levels at the end of acquisition training (irrespective of whether the cortisol levels were endogenously generated or experimentally induced). To determine whether this hormonal memory relationship was sexually dimorphic, we subdivided the high cortisol group by sex. Pearson correlation coefficients did not reveal a significant correlation between post-
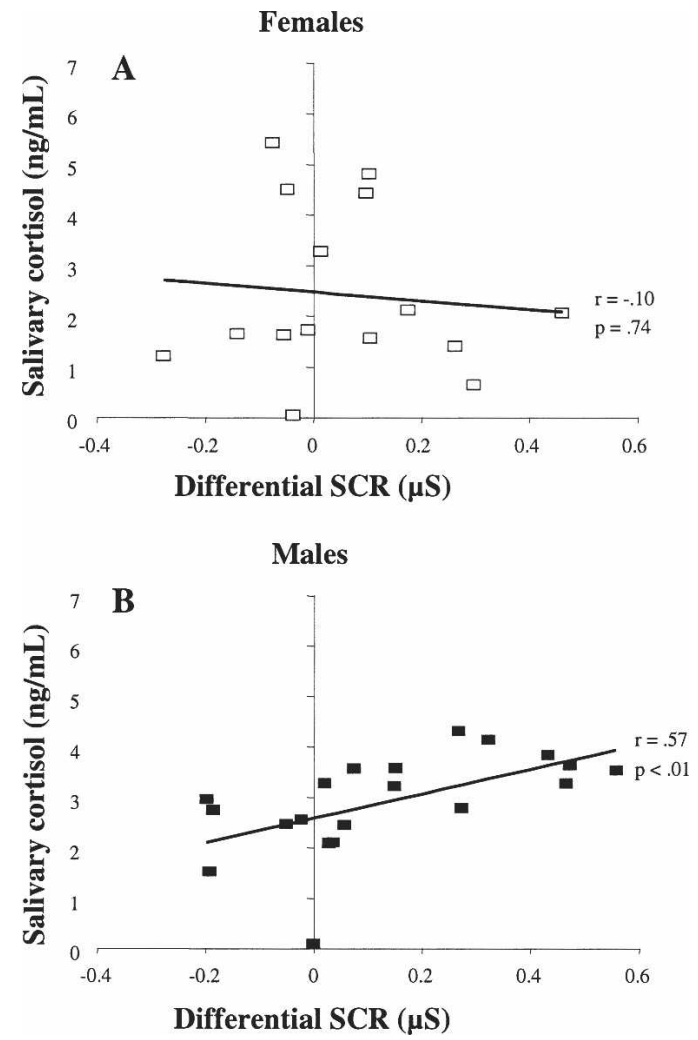

Figure 3. Correlation between differential skin conductance responses (SCRs), expressed as the difference between CS+ and CS - , during late acquisition of conditioning and post-acquisition salivary cortisol levels (+45 min) in female $(A)$ and male $(B)$ participants.

\section{Learning \& Memory}


acquisition cortisol and conditioned fear retention for females $(P=0.39)$, but there was a trend for significance in males $(P=0.06)$. However, the evidence is not sufficient to permit a sex-specific interpretation of this relationship, perhaps due to insufficient statistical power. The possibility that the pattern of results arose from carry-over effects from acquisition to retention in highcortisol males was investigated by correlating acquisition and retention levels in these individuals, but no correlation was found $(P=0.19)$.

\section{Discussion}

The current study investigated the role of sex, psychosocial stress, and cortisol in the acquisition and consolidation of fear conditioning. We found a positive relationship between post-acquisition salivary cortisol levels (reflecting levels present at the end of training) and acquisition of fear learning in male but not female participants. This relationship was not attributable to sex differences in US responsivity, baseline cortisol levels, or subjective measures of the study's aversiveness. Furthermore, we observed a positive relationship between post-acquisition salivary cortisol levels and fear memory consolidation over a 24-h period but only in those participants whose fear learning was initially accompanied by high stress hormone levels.

The sexually dimorphic correlation between cortisol levels and acquisition of fear conditioning in the current study replicates our previously reported findings (Zorawski et al. 2005) and fits nicely with the existing animal literature. Wood et al. (2001) reported that corticosterone levels correlated with fear conditioning in male but not female rats, and Shors et al. (1992) found that stress enhanced subsequent fear conditioning in male rats, whereas the opposite held in female rats. The latter finding was reversed by treatments disabling the estrogen system, such as ovarectomy or administration of estrogen antagonists (Wood and Shors 1998). The beneficial effects of cortisol on fear learning may therefore be affected by estrogen, suggesting complex hormonal interactions in the modulation of emotional memory. Recent research in humans also supports the sex-specific results. Jackson et al. (2006) found that psychosocial stress induced prior to fear conditioning enhanced learning in males but impaired learning in females. Collectively, the fear conditioning data complement other research showing sex differences in amygdala activation and emotional memory modulation (for review, see Cahill 2005).

While our interpretation of the results fits well with the animal literature and theoretical models of the role of glucocorticoids in cognitive processes, putatively involving sexually dimorphic mechanisms (e.g., Oitzl and de Kloet 1992; Shors et al. 1992; Sandi and Rose 1994; Lupien and McEwen 1997; Oitzl et al. 1997; Shors 2001; Wood et al. 2001; Lupien et al. 2002a), we should nevertheless point out that correlational data do not permit conclusions regarding causality and directionality. For example, we cannot rule out the possibility that stronger aversive reactions to the conditioning procedure caused both better acquisition and higher cortisol levels after training. However, we note that individual differences in cortisol levels were relatively stable-the cortisol levels taken at baseline correlated positively with the post-acquisition levels within subjects in both the current study and Zorawski et al. (2005) (both Ps $<0.01$ ). Furthermore, the reported subjective level of irritation from the shock did not correlate either with acquisition of conditioning or with post-acquisition cortisol levels in either males or females (all Ps $>0.17)$.

It may be surprising that overall cortisol levels were not elevated between baseline and post-acquisition time points. This result replicates previous studies of hormones and fear conditioning in humans (Jackson et al. 2006; Zorawski et al. 2005). The diurnal fluctuation in cortisol levels is relatively large and may mask task-induced effects, especially for experiments that are run in the afternoon when levels are naturally declining over time. We note that there was an increase from Sample 3 to Sample 4 in the present study (Fig. 4), which shows a relative increase in cortisol toward the end of training/stress induction. Because of the sluggishness of the hormonal response, it is difficult to specify a tighter coupling of cortisol levels across different points in training where individuals may vary with respect to peak learning or stress induction. Consequently, it was not possible to distinguish the relative contributions of the fear learning acquired late in acquisition training from the psychosocial stress induction in mediating the post-acquisition stress hormone levels. Nonetheless the sex-specific correlation with fear acquisition is similar to that found in Zorawski et al. (2005) where no psychosocial stress was induced. Finally, due to ethical constraints the amount of fear elicited in the laboratory is limited to a range where participants report feeling aversive but not painful reactions, which underestimates fear elicitation in real-world settings or some animal models.

The other key finding of the current study was the association between post-acquisition cortisol and fear memory consolidation. Numerous fear conditioning studies in animals and declarative memory studies in humans have proposed a role for glucocorticoids in the consolidation of emotional memory (Flood et al. 1978; Oitzl and de Kloet 1992; Sandi and Rose 1994; Roozendaal and McGaugh 1996; Buchanan and Lovallo 2001; Zorawski and Killcross 2002, 2003; Abercrombie et al. 2003; Cahill 

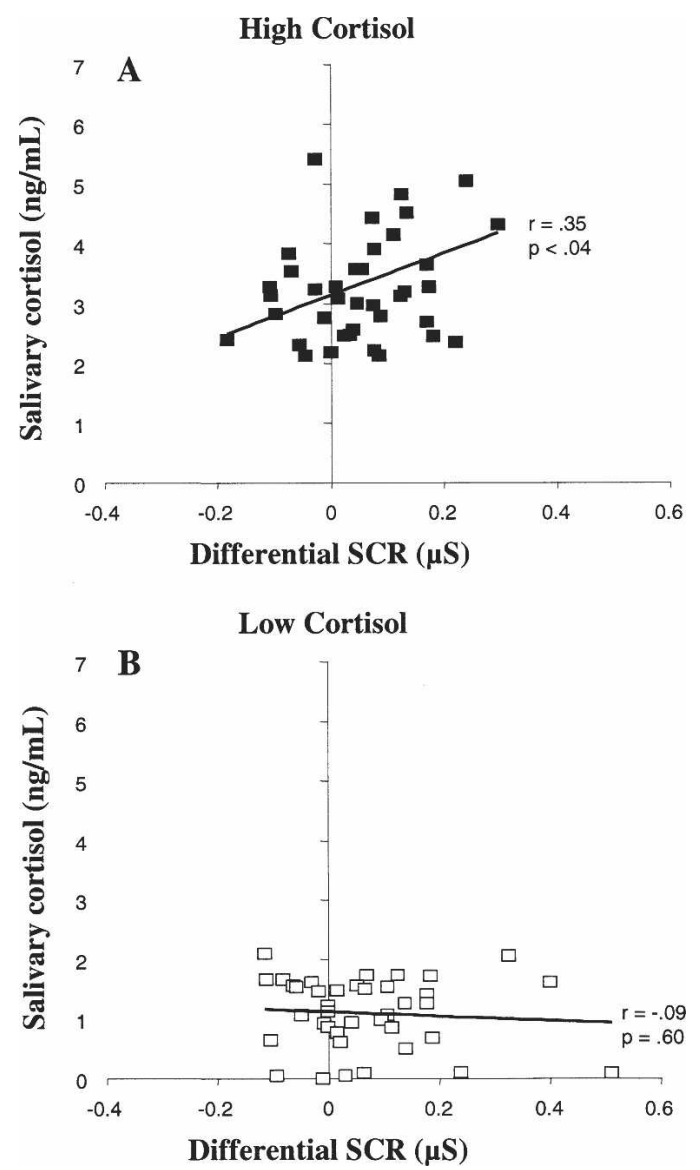

Figure 5. Correlation between differential skin conductance responses (SCRs), expressed as the difference between $\mathrm{CS}+$ and $\mathrm{CS}-$, during the 24-h delayed retention test on Day 2 (reflecting memory consolidation) and post-acquisition salivary cortisol levels on Day 1 (+45 min) in high$(A)$ and low-cortisol $(B)$ participants. Data from participants in both experiments (current study and Zorawski et al. [2005]) were pooled together and assigned to high- and low-cortisol groups according to a median split of post-acquisition salivary cortisol levels ( +45 min on Day 1$)$.

et al. 2004; Hui et al. 2004; Maheu et al. 2004). However, glucocorticoids only become related to memory consolidation once a certain stress threshold is reached. In the current report, the cortisolmemory consolidation relationship held only for those individuals who had high stress levels at the time of initial learning and was observed only after pooling data together with that of our previous study (Zorawski et al. 2005). Most, if not all, of the existent studies have indeed employed salient stress procedures or significant pharmacological manipulations of stress hormone levels in order to observe consolidation effects. While our reported correlation did not show statistically significant sex differences, there was a trend for an effect when the analyses were restricted to males only. It is possible that the sex difference in the cortisol-memory consolidation relationship is relatively weaker than that for the cortisol-fear acquisition relationship, although we note that the former effect had lower statistical power (due to the fact that it is only observed in highstress individuals). Future studies should utilize procedures that produce even higher levels of cortisol, for example, by conducting the research in the morning at the diurnal peak of cortisol levels or by experimental administration of stress hormones. Nonetheless, the current results show that the relationship between high cortisol levels and enhanced memory consolidation extends to the domain of human fear conditioning.
We believe that both of the principal findings can be accounted for by putative differential roles of MRs and GRs in cortisol effects on learning and memory (Lupien and McEwen 1997; Oitzl et al. 1997; Zorawski et al. 2005). MRs are largely occupied under low stress conditions whereas GRs typically become activated under more stressful situations (Reul and de Kloet 1985; de Kloet et al. 1993; Reul et al. 2000). The relative MR/GR occupation ratio of an individual participant would depend on basal cortisol levels and HPA reactivity on the one hand, and the salience of the conditioning and/or psychosocial stress procedures on the other. MRs have been suggested to mediate nonspecific aspects of memory acquisition, such as sensory integration and selective attention (Oitzl and de Kloet 1992; Sandi and Rose 1994; Lupien and McEwen 1997; Oitzl et al. 1997; Lupien et al. 2002a). Since the conditioning procedure itself did not produce any mean increase in cortisol levels (Fig. 4), and since all testing occurred in the afternoon when natural diurnal cortisol is typically low, it is possible that the observed relationship between post-acquisition cortisol levels and acquisition of fear learning was mainly mediated by MRs, with increased occupation leading to increased sensory integration or selective attention, and consequently better fear acquisition (Zorawski et al. 2005). The sexspecificity of this relationship, which occurred in males only, may be due to estrogen selectively affecting MR affinity and availability in females (Wood et al. 2001).

However, when post-acquisition cortisol levels reached a particular threshold, GRs may have become increasingly occupied. As GRs have been specifically implicated in memory consolidation processes (Oitzl and de Kloet 1992; Sandi and Rose 1994; Zorawski and Killcross 2002; Roozendaal 2003), high stress at the time of learning could have translated into a relationship between GR occupation levels and memory consolidation. We therefore hypothesize that only within the cortisol level range that involves GR activation would higher cortisol levels predict the amount of delayed retention. Future research should more thoroughly test whether estrogen mediates the relationship between GR activation and consolidation of fear memories.

Finally, the current results may have important clinical implications. Many neuropsychiatric syndromes have been linked to abnormal emotional learning and memory processes, including anxiety (Öhman 1979; Rosen and Schulkin 1998), posttraumatic stress disorder (Brewin 2001; Layton and Krikorian 2002; Orr et al. 2002), and drug addiction (Everitt et al. 2001; Robinson and Berridge 2003). Abnormal stress hormone levels and stress reactivity may constitute a pathopsychological mechanism of maladaptive emotional learning and thereby contribute to the occurrence of such conditions. Interestingly, some of these conditions have different prevalence rates for men and women (Seedat and Stein 2000; Breslau 2002; Bryant and Harvey 2003). Complex interactions among sex, stress, and fear, and their hormonal and neurobiological bases, are likely to underlie individual differences in fear learning and to mediate the development of affective disorders. A better understanding of these factors may therefore prove instrumental in improving prevention and treatment strategies.

\section{Conclusions}

Fear conditioning constitutes a vital survival mechanism that allows organisms to rapidly learn about emotional events in their environment and adaptively organize their behavior accordingly. Maladaptive variation in fear learning abilities, however, may contribute to the development of psychopathological syndromes such as anxiety, post-traumatic stress disorder, and addiction. Stress hormones, such as cortisol, play an important role in modulating various aspects of fear conditioning, and some of 
these relationships may be sexually dimorphic. A better understanding of the complex neurohormonal interactions among sex, stress, and fear may lead to better prevention and treatment of various forms of psychopathology. This paper contributes to this area of research by reporting a replicable, sexually dimorphic short-term relationship between cortisol levels and fear acquisition, and a long-term relationship between cortisol levels and memory consolidation for individuals whose learning is accompanied by high stress.

\section{Materials and Methods}

\section{Participants}

Fifty-six healthy adults were recruited via advertising at Duke University and were compensated for their participation. Of these, 35 participants (15 females) aged 18-38 years (males: $M=24.74, S D=6.16$; females: $M=22.87, S D=3.89$ ) were included in the study. Given the fear-relevant nature of the conditioned stimuli, participants completed a questionnaire assessing attitudes towards snakes and spiders (Klorman et al. 1974). Individuals scoring within one $S D$ of the mean of phobics (Fredrikson 1983) were excluded from analysis, as in Zorawski et al. (2005). This exclusion criterion was established to provide a homogeneous, healthy sample that did not mix phobic and nonphobic individuals. Additional exclusion criteria included excessively low responsiveness to the US, or any self-reported history of psychiatric or neurological disorders, alcoholism, or substance abuse. In addition, participants were omitted due to technical failure or problems with the cortisol analysis. In order to increase the statistical power of some analyses, data from the current experiment were furthermore pooled with data from our previous study (Zorawski et al. 2005), resulting in a total sample size of 79 (36 females). All participants provided written informed consent that had been approved by the Duke University Institutional Review Board.

\section{Procedure and fear conditioning protocol}

Participants underwent a differential fear conditioning paradigm, as previously described by Zorawski et al. (2005). A picture of a snake and a picture of a spider obtained from the International Affective Picture System (Lang et al. 2001) served as the conditioned stimuli (CS). For each participant, one of the pictures served as the partially reinforced stimulus (CS+) while the other was not reinforced (CS-). Each stimulus was presented centrally on a 17 -inch computer screen $(22.5 \mathrm{~cm} \times 21.5 \mathrm{~cm}$; duration: $4 \mathrm{sec}$ ), in one of two pseudorandom sequences (inter-trial interval: $14 \pm 2 \mathrm{sec}$ ). The designation of pictures as CS+ and CS and the sequence presentation were randomized and counterbalanced across all participants.

An electric shock served as the US. The shock was delivered through an electrode $(21 \mathrm{~mm}$ electrode spacing, Grass-Telefactor model F-E10S2) placed over the median nerve of the participants' dominant wrist. The electrode leads were secured by a rubber strap attached to a Grass-Telefactor SD-9 stimulator by way of coaxial cable leads that were shielded and grounded through a radiofrequency filter. Saline-based gel was used as a conductive electrolyte (Parker Laboratories). The shock was $200 \mathrm{~ms}$ in duration and coterminated with the CS+. Prior to conditioning, shock level intensity was determined individually for each participant, being regarded as unpleasant and aversive but not painful.

The experiment consisted of two sessions separated by a 24-h period. On the first day, participants provided signed consent to participate in the study and were asked to fill out questionnaires which assessed their attitudes towards snakes and spiders as well as their medical history. Prior to conditioning and in order to put participants in a comparable, relaxed state, they were shown a neutral film clip of a scenic train ride through British Columbia (Highball Productions). Following the clip, participants underwent habituation and acquisition consecutively without a break. The habituation phase consisted of four nonreinforced trials of each CS type. The acquisition phase consisted of
16 trials of each CS type. Five of the 16 CS+ trials were reinforced by cotermination with the US, while CS trials were always unreinforced. Following this procedure, participants reported their subjective experience using 7-point scales, assessing the emotionality of the images presented in the study, the irritation caused by the shock, and the general stressfulness of the procedure. Participants then underwent a psychosocial stress procedure (see below).

On Day 2, participants returned to the lab during the same hours as on Day 1 (2 pm-6 pm) and filled out the Daily Stress Inventory (DSI) to assess the degree of stress they experienced in the last $24 \mathrm{~h}$ (Brantley et al. 1987). They then underwent an extinction test consisting of eight non-reinforced, pseudorandomized presentations of each CS type. Participants were unaware that they would not receive shocks during this session and were connected to the stimulating electrode in the same way as on Day 1 . At the end of the session, participants were fully debriefed about the purpose of the experiment and were compensated for their participation.

\section{Task instructions for fear conditioning}

Prior to conditioning and extinction, participants were informed that they would be shown pictures of a snake and a spider and that they might receive occasional shocks. In order to keep participants attentive, they were instructed to classify each picture as a snake or spider with their dominant hand, using the number pad of the keyboard. They were also asked to keep their nondominant hand still to avoid movement artifacts in the SCR recordings.

\section{Physiologic measurements}

SCR was assessed continuously throughout testing and constituted the dependent measure of learning. $\mathrm{Ag}-\mathrm{AgCl}$ electrodes were filled with Sigma Gel as a conductive electrolyte and placed on the middle phalanges of the second and third digits of each participant's nondominant hand (BIOPAC Systems). The responses were monitored at $250 \mathrm{~Hz}$ and stored offline using AcqKnowledge Software for subsequent analysis (BIOPAC Systems). The physiologic data were scored according to conventional methods, as previously described (LaBar et al. 1998, 2004).

\section{Cortisol protocol}

Cortisol was measured in saliva and collection procedures were identical to those reported in Zorawski et al. (2005). All sessions were conducted in the afternoon (approximately 2 p.m. -6 p.m.) when endogenous levels are typically low, to control for the natural diurnal rhythm of cortisol levels. Participants were asked to refrain from exercise, eating, drinking, chewing gum, and smoking for $30 \mathrm{~min}$ prior to the study, as these variables may obscure cortisol measurements. Cortisol samples were obtained at various time points throughout the study. Time points were chosen in consideration of the temporal dynamics of cortisol's entry into saliva by passive diffusion and the fact that saliva cortisol levels typically peak $\sim 30$ min after a stressor (Kirschbaum and Hellhammer 1994). On Day 1, samples were taken $\sim 15 \mathrm{~min}$ before (baseline) and 15, 30, and $45 \mathrm{~min}$ after the beginning of conditioning; on Day 2, samples were taken $\sim 5$ min prior to and $30 \mathrm{~min}$ after the onset of extinction.

For saliva collection, participants placed a cotton dental swab, a Salivette (Sarstedt), in their mouth for $\sim 30$ sec. Samples were labeled and refrigerated until assayed. It has been shown in numerous studies that saliva cortisol levels approximate free, nonprotein-bound cortisol, that the collection method (stimulated or nonstimulated) only affects the volume collected but not the cortisol concentration (Riad-Fahmy et al. 1982; Kirschbaum and Hellhammer 1994), and that cotton-swabbing constitutes a reliable and accurate measurement of cortisol (Shirtcliff et al. 2001). All of the cortisol samples were subjected to freeze thawing centrifugation and measured by ELISA (Oxford Biomedical Research). The sensitivity of the assay was $0.05 \mathrm{ng} / \mathrm{mL}$. Standard, low, and high controls were run in every assay. Inter- and intraassay coefficient of variation on the assay was $<10 \%$ and $5 \%$, respectively. 


\section{Psychosocial stress}

An 11-min psychosocial stress procedure was employed directly following conditioning on Day 1 . The procedure occurred in the same room as conditioning and the electrodes were not removed for this portion of the experiment. The procedure consisted of the paced auditory serial-addition test (PASAT; Gronwall 1977) followed by public speaking. Participants were told that it is important that they take the tasks seriously, do as well as possible, and that their performance would be compared against that of their peers. The PASAT consisted of a 7-min arithmetic task in which single-digit numbers flashed on the screen. At each number presentation, participants were required to add the current number to its predecessor. Participants indicated these sums on a number pad on the screen using a computer mouse. A loud noise burst was delivered over headphones immediately after each incorrect response. The PASAT was designed in such a way that the frequency of number presentation increases rapidly, making it virtually impossible to give correct responses during the latter portion of the task. Upon conclusion of the PASAT, participants were provided with false feedback, indicating extremely poor performance relative to their peers.

Directly following the conclusion of the PASAT, participants completed a 4-min public speech task. Participants were knowingly video-taped for this portion of the task and were misinformed that their video would be sent to the principal investigator for evaluation. The content of their public speech consisted of answering questions regarding their performance on the PASAT, how their performance relates to their performance in other activities of their everyday lives, and whether their performance reflects their general intellectual ability. The timing of the psychosocial stress procedure allowed for saliva sample collections at time points comparable to those used by Zorawski et al. (2005). At the end of the session, participants were informed that the arithmetic and the public speech task were not true measurements of their ability and were designed for the sole purpose of inducing stress.

\section{Data analysis}

Data were analyzed as described in Zorawski et al. (2005). Prior to data analysis, conditioned SCRs were square-root-transformed and range-corrected (LaBar et al. 1995), to normalize the distribution, and collapsed into "early" and "late" trial blocks for habituation and acquisition. Each block consisted of the mean of two trials per CS type for the habituation phase and the mean of eight trials per CS type for the acquisition phase. Extinction trials were not collapsed into early and late blocks, so these data reflected the mean of eight trials per CS type. Conditioning-related changes were hypothesized to occur in the late acquisition phase (LaBar et al. 1995, 2004) while retention performance in the 24-h delayed extinction test was taken as a measure of consolidation of fear learning. Differential SCR scores (CS+ minus CS - ) were also calculated across trials as an index of learning. These difference scores were used to investigate the relationship with posttraining cortisol levels, using median-split and Pearson correlation coefficient methods. Data were analyzed using mixed analyses of variance (ANOVA), with post hoc Bonferroni-corrected $t$-tests as necessary. The $\alpha$ level was set at 0.05 in all analyses.

\section{Acknowledgments}

We thank Lisa Gatti and Judy Johnston for their help with subject recruitment and cortisol analysis, respectively. Supported by NIH grant R01 DA14094 and NSF CAREER Award 0239614.

\section{References}

Abercrombie, H.C., Kalin, N.H., Thurow, M.E., Rosenkranz, M.A., and Davidson, R.J. 2003. Cortisol variation in humans affects memory for emotionally laden and neutral information. Behav. Neurosci. 117: $505-516$

Akirav, I., Kozenicky, M., Tal, D., Sandi, C., Venero, C., and Richter-Levin, G. 2004. A facilitative role for corticosterone in the acquisition of a spatial task under moderate stress. Learn. Mem. 11: $188-195$.
Arbel, I., Kadar, T., Silbermann, M., and Levt, A. 1994. The effects of long-term corticosterone administration on hippocampal morphology and cognitive performance of middle-aged rats. Brain Res. 657: 227-235.

Bartels, M., De Geus, E.J.C., Kirschbaum, C., Sluyter, F., and Boomsma D.I. 2003. Heritability of daytime cortisol levels in children. Behav. Genet. 33: 421-433.

Beckwith, B.E., Petros, T.V., Scaglione, C., and Nelson, J. 1986 Dose-dependent effects of hydrocortisone on memory in human males. Physiol. Behav. 36: 283-286.

Bemelsmans, K.J., Goekoop, J.G., De Rijk, R., and Van Kempen, G.M.J. 2002. Recall performance, plasma cortisol and plasma norepinephrine in normal human subjects. Biol. Psychol. 62: 1-15.

Bodnoff, S.R., Humphreys, A.G., Lehman, J.C., Diamond, D.M., Rose, G.M., and Meaney, M.J. 1995. Enduring effects of chronic corticosterone treatment on spatial learning, synaptic plasticity, and hippocampal neuropathology in young and mid-aged rats. $J$. Neurosci. 15: 61-69.

Brantley, P.J., Waggoner, C.D., Jones, G.N., and Rappaport, N.B. 1987. A daily stress inventory: Development, reliability, and validity. $J$. Behav. Med. 10: 61-74.

Breslau, N. 2002. Gender differences in trauma and posttraumatic stress disorder. J. Gend. Specif. Med. 5: 34-40.

Brewin, C.R. 2001. A cognitive neuroscience account of posttraumatic stress disorder and its treatment. Behav. Res. Ther. 39: 373-393.

Bryant, R.A. and Harvey, A.G. 2003. Gender differences in the relationship between acute stress disorder and posttraumatic stress disorder following motor vehicle accidents. Aust. N. Z. J. Psychiatry 37: 226-229.

Buchanan, T.W. and Lovallo, W.R. 2001. Enhanced memory for emotional material following stress-level cortisol treatment in humans. Psychoneuroendocrinology 26: 307-317.

Buss, C., Wolf, O.C., Witt, J., and Hellhammer, D.H. 2004 Autobiographic memory impairment following acute cortisol administration. Psychoneuroendocrinology 29: 1093-1096.

Cahill, L. 2005. His brain, her brain. Sci. Am. 292: 40-47.

Cahill, L., Gorski, L., and Le, K. 2004. Enhanced human memory consolidation with post-learning stress: Interaction with the degree of arousal at encoding. Learn. Mem. 10: 270-274.

Conrad, C.D., Galea, L.A., Kuroda, Y., and McEwen, B.S. 1996. Chronic stress impairs rat spatial memory on the Y maze, and this effect is blocked by tianeptine pretreatment. Behav. Neurosci. 110: $1321-1334$.

Conrad, C.D., Lupien, S.J., and McEwen, B.S. 1999. Support for a bimodal role for Type II adrenal steroid receptors in spatial memory. Neurobiol. Learn. Mem. 72: 39-46.

Cools, A.R. and Gingras, M.A. 1998. Nijmegen high and low responders to novelty: A new tool in the search after the neurobiology of drug abuse liability. Pharmacol. Biochem. Behav. 60: 151-159.

de Kloet, E.R. 2004. Hormones and the stressed brain. Ann. N. Y. Acad. Sci. 1018: $1-15$

de Kloet, E.R., Oitzl, M., and Joels, M. 1993. Functional implications of brain corticosteroid receptor diversity. Cell. Mol. Neurobiol. 13: $433-455$.

De Quervain, D.J.F., Roozendaal, B., and McGaugh, J.L. 1998. Stress and glucocorticoids impair retrieval of long-term spatial memory. Nature 394: 787-790.

De Quervain, D.J.F., Roozendaal, B., Nitsch, R.M., McGaugh, J.L., and Hock, C. 2000. Acute cortisone administration impairs retrieval of long-term declarative memory in humans. Nat. Neurosci. 3: 313-314.

Diamond, D.M., Bennett, M.C., Fleshner, M., and Rose, G. 1992. Inverted-U relationship between the level of peripheral corticosterone and the magnitude of hippocampal primed burst potentiation. Hippocampus 2: 421-430.

Domes, G., Heinrichs, M., Reichwald, U., and Hautzinger, M. 2002. Hypothalamic-pituitary-adrenal axis reactivity to psychological stress and memory in middle-aged women: High responders exhibit enhanced declarative memory performance. Psychoneuroendocrinology 27: 843-853.

Everitt, B.J., Dickinson, A., and Robbins, T.W. 2001. The neuropsychological basis of addictive behaviour. Brain Res. Brain Res. Rev. 36: 129-138.

Fehm-Wolfsdorf, G., Reutter, K., Zenz, H., Born, J., and Fehm, H.L. 1993. Are circadian variations in taste thresholds cortisol-dependent? J. Psychophysiol. 7: 65-72.

Flood, J.F., Vidal, D., Bennett, E.L., Orme, A.E., Vasquez, S., and Jarvik, M.E. 1978. Memory facilitating and anti-amnesic effects of corticosteroids. Pharmacol. Biochem. Behav. 8: 81-87.

Fredrikson, M. 1983. Reliability and validity of some specific fear questionnaires. Scand. J. Psychol. 24: 331-334.

Gronwall, D.M. 1977. Paced auditory serial-addition task: A measure of recovery from conclusion. Percept. Mot. Skills 44: 367-373. 
Hui, G.K., Figueroa, I.R., Poytress, B.S., Roozendaal, B., McGaugh, J.L. and Weinberger, N. 2004. Memory enhancement of classical fear conditioning by post-training injections of corticosterone in rats. Neurobiol. Learn. Mem. 81: 67-74.

Jackson, E.D., Payne, J.D., Nadel, L., and Jacobs, W.J. 2006. Stress differentially modulates fear conditioning in healthy men and women. Biol. Psychiatry 59: 516-522.

Kabbaj, M., Devine, D.P., Savage, V.R., and Akil, H. 2000. Neurobiological correlates of individual differences in novelty-seeking behavior in the rat: Differential expression of stress-related molecules. J. Neurosci. 20: 6983-6988.

Kirschbaum, C. and Hellhammer, D.H. 1994. Salivary cortisol in psychoneuroendocrine research: Recent developments and applications. Psychoneuroendocrinology 19: 313-333.

Kirschbaum, C., Wolf, O.T., May, M., Wippich, W., and Hellhammer D.H. 1996. Stress- and treatment-induced elevations of cortisol levels associated with impaired declarative memory in humans. Life Sci. 58: $1475-1483$.

Kirschbaum, C., Kudielka, B.M., Gaab, J., Schommer, N.C., and Hellhammer, D.H. 1999. Impact of gender, menstrual cycle Phase, and oral contraceptives on the activity of the hypothalamus-pituitary-adrenal Axis. Psychosom. Med. 61: 154-162.

Klorman, R., Hastings, J.E., Weerts, T.C., Melamed, B.G., and Lang, P.J. 1974. Psychometric description of some specific-fear questionnaires. Behav. Ther. 5: 401-409.

Kovacs, G.L., Telegdy, G., and Lissak, K. 1977. Dose-dependent action of corticosteroids on brain serotonin content and passive avoidance behavior. Horm. Behav. 8: 155-165.

LaBar, K.S., LeDoux, J.E., Spencer, D.D., and Phelps, E.A. 1995. Impaired fear conditioning following unilateral temporal lobectomy in humans. J. Neurosci. 15: 6846-6855.

LaBar, K.S., Gatenby, J.C., Gore, J.C., LeDoux, J.E., and Phelps, E.A. 1998. Human amygdala activation during conditioned fear acquisition and extinction: A mixed-trial fMRI study. Neuron 20: $937-945$.

LaBar, K.S., Cook, C.A., Torpey, D.C., and Welsh-Bohmer, K.A. 2004. Impact of healthy aging on awareness and fear conditioning. Behav. Neurosci. 118: 905-915.

Lang, P.J., Bradley, M.M., and Cuthbert, B.N. 2001. International affective picture system (IAPS): Instruction manual and affective ratings. Technical report A-5. University of Florida, Gainesville, FL.

Layton, B. and Krikorian, R. 2002. Memory mechanisms in posttraumatic stress disorder. J. Neuropsychiatry Clin. Neurosci. 14: $254-261$

Luine, V.N., Spencer, R.L., and McEwen, B.S. 1993. Effects of chronic corticosterone ingestion on spatial memory performance and hippocampal serotonergic function. Brain Res. 616: 65-70.

Luine, V.N., Villegas, M., Martinez, C., and McEwen, B.S. 1994. Repeated stress causes reversible impairments of spatial memory performance. Brain Res. 639: 167-170.

Lupien, S.J. and McEwen, B.S. 1997. The acute effects of corticosteroids on cognition: Integration of animal and human model studies. Brain Res. Brain Res. Rev. 24: 1-27.

Lupien, S.J., Gaudreau, S., Tchiteya, B.M., Maheu, F., Sharma, S., Nair, N.P., Hauger, R.L., McEwen, B.S., and Meaney, M.J. 1997. Stress-induced declarative memory impairment in healthy elderly subjects: Relationship to cortisol reactivity. J. Clin. Endocrinol. Metab. 82: 2070-2075.

Lupien, S.J., Gillin, C., and Hauger, R.L. 1999. Working memory is more sensitive than declarative memory to the acute effects of corticosteroids: A dose-response study. Behav. Neurosci. 13: 1-11.

Lupien, S.J., Wilkinson, C.W., Brière, S., Mènard, C., Ng Ying Kin, N.M.K., and Nair, N.P.V. 2002a. The modulatory effects of corticosteroids on cognition: Studies in young human populations. Psychoneuroendocrinology 27: 401-416.

Lupien, S.J., Wilkinson, C.W., Brière, S., Ng Ying Kin, N.M.K., Meaney, M.J., and Nair, N.P.V. 2002b. Acute manipulation of aged human memory by pharmacological manipulation of glucocorticoids. $J$. Clin. Endocrinol. Metab. 87: 3798-3807.

Maheu, F.S., Joober, R., Beaulieu, S., and Lupien, S.J. 2004. Differential effects of adrenergic and corticosteroid hormonal systems on human short- and long-term declarative memory for emotionally arousing material. Behav. Neurosci. 118: 420-428.

Maheu, F.S., Collicutt, P., Kornik, R., Mozkowski, R., and Lupien, S.J. 2005a. The perfect time to be stressed: A differential modulation of human memory by stress applied in the morning or in the afternoon. Prog. Neuropsychopharmacol. Biol. Psychiatry 29: $1281-1288$.

Maheu, F.S., Joober, R., and Lupien, S.J. 2005b. Declarative memory after stress in humans: Differential involvement of the $\beta$-adrenergic and corticosteroid systems. J. Clin. Endocrinol. Metab. 90: 1697-1704

McEwen, B.S. and Sapolsky, R.M. 1995. Stress and cognitive function.
Curr. Opin. Neurobiol. 5: 205-216.

McGaugh, J.L. 2004. The amygdala modulates the consolidation of memories of emotionally arousing experiences. Annu. Rev. Neurosci. 27: 1-28.

Meaney, M.J., Bhatnagar, S., Larocque, S., McCormick, C., Shanks, N., Sharn, S., Smythe, J., Viau, V., and Plotsky, P.M. 1993. Individual differences in the hypothalamic-pituitary-adrenal stress response and the hypothalamic CRF system. Ann. N. Y. Acad. Sci. 697: 70-85.

Newcomer, J.W., Selke, G., Melson, A.K., Hershey, T., Craft, S., Richards, K., and Alderson, A.L. 1999. Decreased memory performance in healthy humans induced by stress-level cortisol treatment. Arch. Gen. Psychiatry 56: 527-533.

Öhman, A. 1979. Fear relevance, autonomic conditioning, and phobias: A laboratory model. In Trends in behavior therapy (eds. P.-O. Sjödénm et al.), pp. 107-134. Academic Press, New York.

Oitzl, M.S. and de Kloet, E.R. 1992. Selective corticosteroid antagonists modulate specific aspects of spatial orientation learning. Behav. Neurosci. 106: 62-71.

Oitzl, M.S., van Haarst, A.D., and de Kloet, E.R. 1997. Behavioral and neuroendocrine responses controlled by the concerted action of central mineralocorticoid (MRs) and glucocorticoid receptors (GRs). Psychoneuroendocrinology 22: S87-S93.

Orr, S.P., Metzger, L.J., and Pitman, R.K. 2002. Psychophysiology of post-traumatic stress disorder. Psychiatr. Clin. North Am. 25: 271-293.

Park, C.R., Campbell, A.M., and Diamond, D.M. 2001. Chronic psychosocial stress impairs learning and memory and increases sensitivity to yohimbine in adult rats. Biol. Psychiatry 50: 994-1004.

Piazza, P.V., Maccari, S., Deminiere, J.M., Le Moal, M., Mormede, P., and Simon, H. 1991. Corticosterone levels determine individual vulnerability to amphetamine self-administration. Proc. Natl. Acad. Sci. 88: 2088-2092.

Putman, P., Van Honk, J., Kessels, R.P.C., Mulder, M., and Koppeschaar, H.P.F. 2004. Salivary cortisol and short and long-term memory for emotional faces in healthy young women. Psychoneuroendocrinology 29: 953-960.

Reul, J.M. and de Kloet, E.R. 1985. Two receptor systems for corticosterone in rat brain: Microdistribution and differential occupation. Endocrinology 117: 2505-2511.

Reul, J.M., Gesing, A., Droste, S., Stec, I.S., Weber, A., Bachmann, C. Bilang-Bleuel, A., Holsboer, F., and Linthorst, A.C. 2000. The brain mineralocorticoid receptor: Greedy for ligand, mysterious in function. Eur. J. Pharmacol. 405: 235-249.

Riad-Fahmy, G., Read, F., Walker, R.F., and Griffiths, K. 1982. Steroids in saliva for assessing endocrine function. Endocr. Rev. 3: 367-395.

Robinson, T.E. and Berridge, K.C. 2003. Addiction. Annu. Rev. Psychol. 54: $25-53$.

Rohleder, N., Wolf, J.M., and Kirschbaum, C. 2003. Glucocorticoid sensitivity in humans-interindividual differences and acute stress effects. Stress 6: 207-222.

Roozendaal, B. 2003. Systems mediating acute glucocorticoid effects on memory consolidation and retrieval. Prog. Neuropsychopharmacol. Biol. Psychiatry 27: 1213-1223.

Roozendaal, B. and McGaugh, J.L. 1996. Amygdaloid nuclei lesions differentially affect glucocorticoid induced memory enhancement in an inhibitory avoidance task. Neurobiol. Learn. Mem. 65: 1-8.

Rosen, J.B. and Schulkin, J. 1998. From normal fear to pathological anxiety. Psychol. Rev. 105: 325-350.

Sandi, C. and Rose, S.P.R. 1994. Corticosterone enhances long-term retention in one-day-old chicks trained in a weak passive avoidance learning paradigm. Brain Res. 647: 106-112.

Seedat, S. and Stein, D.J. 2000. Trauma and post-traumatic stress disorder in women: A review. Int. Clin. Psychopharmacol. 15: $\mathrm{S} 25-\mathrm{S} 33$.

Shirtcliff, E.A., Granger, D.A., Schwartz, E., and Curran, M.J. 2001. Use of salivary biomarkers in biobehavioral research: Cotton-based sample collection methods can interfere with salivary immunoassay results. Psychoneuroendocrinology 26: 165-173.

Shors, T.J. 2001. Acute stress rapidly and persistently enhances memory formation in the male rat. Neurobiol. Learn. Mem. 75: 10-29. .2004. Learning during stressful times. Learn. Mem. 11: 137-144.

Shors, T.J., Weiss, C., and Thompson, R.F. 1992. Stress-induced facilitation of classical conditioning. Science 257: 537-539.

Starkman, M.N., Gebarski, S.S., Berent, S., and Schteingart, D.E. 1992. Hippocampal formation volume, memory dysfunction, and cortisol levels in patients with Cushing's syndrome. Biol. Psychiatry 32: 756-765.

Steptoe, A., Kunz-Ebrecht, S., Owen, N., Feldman, P.J., Willemsen, G. Kirschbaum, C., and Marmot, M. 2003. Socioeconomic status and stress-related biological responses over the working day. Psychosom. Med. 65: 461-470.

Stone, A.A., Schwartz, J.E., Smyth, J., Kirschbaum, C., Cohen, S., and Hellhammer, X. 2001. Individual differences in the diurnal cycle of 
salivary free cortisol: A replication of flattened cycles for some individuals. Psychoneuroendocrinoloy 26: 295-306.

Veldhuis, H.D., Van Koppen, C., Van Ittersum, M., and de Kloet, E.R. 1982. Specificity of the adrenal steroid receptor system in rat hippocampus. Endocrinology 110: 2044-2051.

Wilson, L.M., Wilson, J.R., and Dicara, L.V. 1975. Facilitation of Pavlovian conditioned cardiodecelerations following preshock in immobilized rats. Physiol. Behav. 15: 653-658.

Wolf, O.T. 2003. HPA axis and memory. Best Pract. Res. Endocrinol. Metab. 17: 287-299.

Wolf, O.T., Convit, A., McHugh, P.F., Kandil, E., Thorn, E.L., De Santi, S., McEwen, B.S., and de Leon, M.J. 2001a. Cortisol differentially affects memory in young and elderly men. Behav. Neurosci. 115: $1002-1011$.

Wolf, O.T., Schommer, N.C., Hellhammer, D.H., McEwen, B.S., and Kirschbaum, C. 2001b. The relationship between stress induced cortisol levels and memory differs between men and women. Psychoneuroendocrinology 26: 711-720.

Wolkowitz, O.M., Reus, V.I., Weingartner, H., Thompson, K., Breier, A., Doran, A., Rubinow, D., and Pickar, D. 1990. Cognitive effects of corticosteroids. Am. J. Psychiatry 147: 1297-1303.

Wood, G.E. and Shors, T.J. 1998. Stress facilitates classical conditioning in males, but impairs classical conditioning in females through activational effects of ovarian hormones. Proc. Natl. Acad. Sci. 95: 4066-4071.

Wood, G.E., Beylin, A.V., and Shors, T.J. 2001. The contribution of adrenal and reproductive hormones to the opposing effects of stress on trace conditioning in males versus females. Behav. Neurosci. 115: $175-187$.

Zorawski, M. and Killcross, S. 2002. Post-training glucocorticoid receptor agonist enhances memory in appetitive and aversive Pavlovian discrete-cue conditioning paradigms. Neurobiol. Learn. Mem. 78: $458-464$.

.2003. Glucocorticoid receptor agonist enhances Pavlovian appetitive conditioning but disrupts outcome-specific associations. Behav. Neurosci. 117: 1453-1457.

Zorawski, M., Cook, C.A., Kuhn, C.M., and LaBar, K.S. 2005. Sex, stress, and fear: Individual differences in conditioned learning. Cogn. Affect. Behav. Neurosci. 5: 191-201.

Received January 16, 2006; accepted in revised form April 18, 2006. 


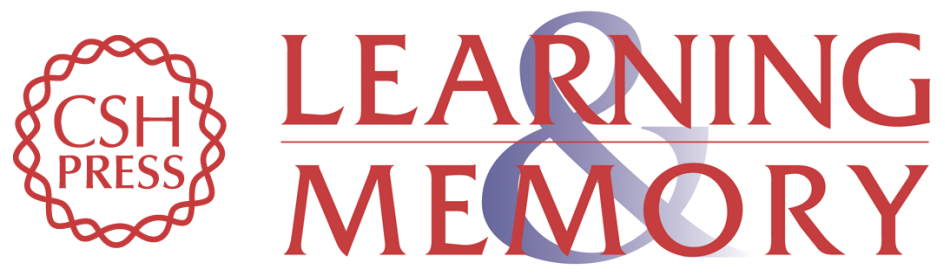

\section{Effects of stress and sex on acquisition and consolidation of human fear conditioning}

Michael Zorawski, Nineequa Q. Blanding, Cynthia M. Kuhn, et al.

Learn. Mem. 2006, 13:

Access the most recent version at doi:10.1101//m.189106

References This article cites 89 articles, 10 of which can be accessed free at:

http://learnmem.cshlp.org/content/13/4/441.full.html\#ref-list-1

License

Email Alerting Receive free email alerts when new articles cite this article - sign up in the box at the Service top right corner of the article or click here. 